\title{
Clinicopathological characteristics and prognostic factors of gastrointestinal stromal tumors in Chinese patients
}

\author{
MING-LEI YANG $^{1 *}, \mathrm{JUN}^{*}$ CHENG WANG ${ }^{1 *},{\mathrm{WEN}-\mathrm{BIN} \mathrm{ZOU}^{2} \text { and DING-KANG YAO }}^{1}$ \\ ${ }^{1}$ Department of Internal Medicine, Changzheng Hospital, Second Military Medical University, Shanghai 200003; \\ ${ }^{2}$ Department of Gastroenterology, Changhai Hospital, Second Military Medical University, Shanghai 200433, P.R. China
}

Received October 27, 2017; Accepted July 11, 2018

DOI: $10.3892 / \mathrm{ol} .2018 .9320$

\begin{abstract}
Gastrointestinal stromal tumors (GISTs) are the most common mesenchymal neoplasms in the gastrointestinal tract, exhibiting wide variability in their biological behavior. The aim of the present study was to investigate the clinicopathological characteristics and prognostic factors of GISTs in Chinese patients. All GIST cases $(n=182)$ retrieved from the pathology database and the archived files in Shanghai Changzheng Hospital between January 2011 and December 2014 were reviewed. The clinical symptoms, preoperative investigations, treatments, pathological characteristics and follow-up data of these patients were reviewed, and univariate and multivariate survival analyses were performed. A total of $73.1 \%$ of the GISTs were located in the stomach, and the most common three symptoms included abdominal pain (30.2\%), dyspepsia (23.1\%) and gastrointestinal bleeding $(21.4 \%)$. Univariate analysis revealed that larger tumor size $(\mathrm{P}<0.001)$, higher mitotic rate $(\mathrm{P}<0.001)$, aggressive behavior $(\mathrm{P}<0.001)$, negative smooth muscle actin expression $(\mathrm{P}=0.009)$ and palliative resection $(\mathrm{P}<0.001)$ contributed toward poor overall survival (OS). In addition, non-gastric disease location $(\mathrm{P}<0.001)$, larger tumor size $(\mathrm{P}<0.001)$, higher mitotic rate $(\mathrm{P}=0.004)$, aggressive behavior $(\mathrm{P}<0.001)$ and palliative resection $(\mathrm{P}<0.001)$ were associated with poor relapse-free survival (RFS). Multivariate analysis indicated that mitotic rate [hazard ratio $(\mathrm{HR}=3.761, \mathrm{P}=0.015)$ ] and aggressive behavior $(\mathrm{HR}=3.916, \mathrm{P}=0.010)$ were independent risk factors for $\mathrm{OS}$, while non-gastric location $(\mathrm{HR}=4.740, \mathrm{P}=0.002)$ and aggressive behavior $(\mathrm{HR}=4.009, \mathrm{P}=0.004)$ were independent risk factors for RFS. The present study provided information
\end{abstract}

Correspondence to: Professor Ding-Kang Yao, Department of Internal Medicine, Changzheng Hospital, Second Military Medical University, 415 Fengyang Road, Shanghai 200003, P.R. China

E-mail: czyaodingkang@163.com

*Contributed equally

Key words: gastrointestinal stromal tumor, clinical symptoms, clinicopathological characteristics, immunohistochemistry, prognosis on the clinicopathological characteristics and epidemiology of GISTs in the Chinese population. Non-gastric disease location, higher mitotic rate and tumor metastasis or local invasion prior to treatment were identified as predictors of a poor prognosis.

\section{Introduction}

Gastrointestinal stromal tumors (GISTs) are the most common mesenchymal tumors of the gastrointestinal tract, accounting for $80 \%$ of all digestive mesenchymal tumors. It is widely accepted that GISTs arise from the interstitial cells of Cajal, and the term 'stromal tumor' was first introduced by Mazur and Clark in 1983 (1). The incidence of GISTs has been reported to range between 11 and 15 per million annually (2-4), and $60 \%$ of GISTs are located in the stomach, $30 \%$ in the jejunum or ileum, $5 \%$ in the duodenum and $4 \%$ in the colorectum. Extragastrointestinal GISTs (EGISTs) have been reported in the liver, omentum, mesentery, gallbladder and urinary bladder (5-7).

The diagnosis of GISTs is based on morphology, positive immunohistochemistry (IHC) results for CD117 and DOG1, and mutation analyses of KIT and platelet-derived growth factor receptor $\alpha$ polypeptide gene (PDGFRA) (7-9). With increasing use of abdominal computed tomography (CT), magnetic resonance imaging (MRI) and endoscopy, an increasing number of asymptomatic GISTs are diagnosed at an early stage, although the effect of early detection of GIST on the prognosis remains unclear. The National Institutes of Health (NIH) and Armed Forces Institute of Pathology (AFIP) risk classification criteria are commonly used to predict the prognosis of GISTs (10-12). Large tumor size, high mitotic rate, non-gastric tumor location and tumor ulceration are commonly accepted to be associated with a poor prognosis in patients with GIST. Other factors, including sex, age, symptoms and IHC results are also reported to be associated with patient outcomes $(13,14)$. However, the biological behavior of GISTs varies widely, with unclear risk predictors, and it is difficult to predict their malignant potential with the currently available risk classification criteria (15).

The number of studies on the clinicopathological characteristics of GIST in China is limited. The aim of the present study was to update the clinicopathological and immunophenotypic characteristics of GISTs in mainland China, and to investigate the prognostic factors of GISTs based on these patients. 


\section{Materials and methods}

Patients and diagnosis. The present study was approved by the Institutional Review Board of Changzheng Hospital. Written informed consent was obtained from all patients involved for the publication of any associated data and accompanying images. The clinicopathological and survival data of 182 patients with GIST treated surgically at Shanghai Changzheng Hospital between January 2011 and December 2014 were retrospectively reviewed. A total of 94 males and 88 females were included. The mean age of the patients was $59.2 \pm 12.6$ years (range, 26-88 years). The diagnosis of each patient with GIST was established based on the results of the histopathology and IHC. Pathological samples were collected during surgical interventions. If the diagnosis of GIST was uncertain based on pathology, mutation analysis for the KIT and PDGFRA genes was performed. For 136 patients with mitotic rate data, the tumors were categorized into different risk groups according to the modified NIH and AFIP risk classification criteria.

The following details of these patients were collected: Age, sex, symptoms and signs, preoperative investigations, surgical details, pathology and follow-up data. The tumor site was analyzed according to previous classification methods $(16,17)$. Preoperative investigations comprised radiological and endoscopic examinations, including gastroscopy, abdominal CT, MRI, colonoscopy, small intestinal endoscopy, capsule endoscopy and positron emission tomography (PET)-CT.

Pathology and IHC. Tissues were fixed in formalin for 12-24 h at room temperature then paraffin-embedded. Sections of $4 \mu \mathrm{M}$ were stained with Hematoxylin \& Eosin at room temperature (3-5 min for hematoxylin and 5-10 sec for eosin) (18). Tissue sections were deparaffined in xylene and rehydrated in a descending alcohol series. Antigen retrieval was performed by heating the sections for $30 \mathrm{~min}$ at $95^{\circ} \mathrm{C}$ in $1 \mathrm{mM}$ EDTA buffer $(\mathrm{pH} 8.0)$. Endogenous peroxidase activity was eliminated by treating sections with 3\% methanolic hydrogen peroxide solution for $10 \mathrm{~min}$. Thereafter, the slides were blocked in 1/100 diluted goat serum (cat. no. kit-9710; Fuzhou Maixin Biotech Co., Ltd., Fuzhou China) for $20 \mathrm{~min}$ at room temperature. IHC analysis included common biomarkers for the diagnosis of GISTs, including CD117 (dilution, 1:400; cat. no. kit-0029; Fuzhou Maixin, Co., Ltd., Fuzhou, China), DOG1 (dilution, 1:400; cat. no. kit-0035; Fuzhou Maixin Biotech Co., Ltd.), CD34 (dilution, 1:600; cat. no. kit-0004; Fuzhou Maixin Biotech Co., Ltd.), smooth muscle actin (SMA) (dilution, 1:600 dilution; cat. no. kit-0006; Fuzhou Maixin Biotech Co., Ltd.), S-100 protein (dilution, 1:500; cat. no. kit-0007; Fuzhou Maixin Biotech Co., Ltd.), Ki-67 (dilution, 1:500; cat. no. kit-0005; Fuzhou Maixin Biotech Co., Ltd.) and desmin (dilution, 1:600; cat. no. kit-0023; Fuzhou Maixin Biotech Co., Ltd.). The sections were incubated with the aforementioned antibodies at $4^{\circ} \mathrm{C}$ overnight. Following 3 washes in PBS, the sections were incubated with biotin-conjugated secondary antibody (ready-to-use; $50 \mu 1$ for each section; goat anti-mouse IgG secondary antibody for CD34, SMA, S-100, Ki-67 and desmin; goat anti-rabbit IgG secondary antibody for CD117 and DOG1) (cat. no. kit-9710; Fuzhou Maixin Biotech Co., Ltd.) for $10 \mathrm{~min}$ at room temperature, followed by incubation with peroxidase-conjugated biotin-streptavidin complex (Fuzhou
Maixin Biotech Co., Ltd.) for $10 \mathrm{~min}$ at room temperature, and finally stained with diaminobenzidine at room temperature for $2 \mathrm{~min}$, and counterstained with hematoxylin at room temperature for 3-5 min. Mitoses were counted in 50 high-power fields (HPF). Two professional pathologists reviewed these results under a light microscope (x100 and x400, magnification).

Treatment methods and follow-up. For localized primary GISTs, radical resection, including open surgical resection and minimally invasive techniques, were selected as the primary treatments (19). Minimally invasive techniques included laparoscopic surgery, endoscopic surgery and endoscopy-assisted laparoscopic surgery $(20,21)$. For locally advanced unresectable or metastatic GISTs, palliative surgery and/or imatinib treatment were recommended, and imatinib adjuvant therapy following radical resection was recommended for patients with intermediate-to-high-risk GISTs (22). The final treatment decision was made with the consent of the patients. The records of all surgical procedures were reviewed. Patient follow-up was conducted by regular hospital visits at 3, 6 and 12 months, and annually thereafter. Each visit included a medical review, physical examination and associated investigations. The patients' status was confirmed by telephone communication at the end of the study. Survival outcomes were assessed in terms of overall survival (OS) and relapse-free survival (RFS). OS was defined as the time from the date of initial treatment to the date of the last follow-up or mortality, and RFS was defined as the time from the date of initial treatment to the time of clinical or radiological evidence of disease relapse or the date of the last follow-up.

Statistical analysis. SAS 9.3 software (SAS Institute, Inc., Cary, NC, USA) and SPSS 23.0 (IBM Corp., Armonk, NY, USA) were used for statistical analysis. Continuous variables are expressed as mean \pm standard deviation and were compared using an unpaired, two-tailed Student's t-test. Categorical variables were compared using the $\chi^{2}$ test or Fisher's exact test. The Kaplan-Meier method and the log-rank test were used for survival analysis. Independent factors were identified in multivariate analysis using the Cox proportional hazard model. $\mathrm{P}<0.05$ was considered to indicate a statistically significant difference.

\section{Results}

Clinicopathological characteristics. A total of 182 patients with GISTs were analyzed in the present study. The stomach was the most common site, accounting for $73.1 \%$ of the cases, followed by the jejunum and ileum (14.3\%), duodenum (5.5\%), colorectum and anus (4.94\%), esophagus (1.1\%), and EGISTs (1.1\%). Abdominal pain, dyspepsia and gastrointestinal bleeding were the main presenting complaints, reported in 30.2, 23.1 and $21.4 \%$ of the patients, respectively. The symptoms varied according to the primary location. Patients with esophageal GISTs often presented with dysphagia (2/2 patients), whereas patients with GISTs of the colorectum or anal canal usually reported altered bowel habits (4/9 patients; Table I).

Gastroscopy and abdominal CT were used in $83(54.6 \%)$ and $87(57.2 \%)$ patients, respectively, with a high diagnostic accuracy of 62.7 and $74.7 \%$, respectively. Other investigations, 
Table I. Clinical characteristics of 182 patients with gastrointestinal stromal tumor.

\begin{tabular}{|c|c|}
\hline Factor & $\mathrm{n}$ \\
\hline \multicolumn{2}{|l|}{ Sex, n $(\%)$} \\
\hline Male & $94(51.6)$ \\
\hline Female & $88(48.4)$ \\
\hline \multicolumn{2}{|l|}{ Age, years } \\
\hline Median & 60 \\
\hline Range & $26-88$ \\
\hline \multicolumn{2}{|l|}{ Location, n (\%) } \\
\hline Esophagus & $2(1.10)$ \\
\hline Stomach & $133(73.1)$ \\
\hline Duodenum & $10(5.50)$ \\
\hline Jejunum and ileum & $26(14.3)$ \\
\hline Colon, rectum and anus & $9(4.94)$ \\
\hline Other (liver and omentum) & $2(1.10)$ \\
\hline \multicolumn{2}{|l|}{ Immunohistochemistry, n (\%) } \\
\hline CD117 & $179(98.4)$ \\
\hline DOG-1 & $177(98.3)$ \\
\hline CD34 & $171(94.5)$ \\
\hline Desmin & $24(13.9)$ \\
\hline S-100 & $25(14.2)$ \\
\hline SMA & $100(57.5)$ \\
\hline \multicolumn{2}{|l|}{ Symptoms, n (\%) } \\
\hline Abdominal pain & $55(30.2)$ \\
\hline Dyspepsia & $42(23.1)$ \\
\hline Gastrointestinal bleeding & $39(21.4)$ \\
\hline Regurgitation & $11(6.04)$ \\
\hline Palpable mass & $8(4.40)$ \\
\hline Altered bowel habit & $8(4.40)$ \\
\hline Weight loss & $4(2.20)$ \\
\hline Fever & $3(1.65)$ \\
\hline Dysphagia & $2(1.10)$ \\
\hline Vomiting & $2(1.10)$ \\
\hline \multicolumn{2}{|c|}{ Pre-operation examinations, $\mathrm{n}(\%)^{\mathrm{a}}$} \\
\hline Gastroscopy & $83(54.6)$ \\
\hline Abdominal CT scan & $87(57.2)$ \\
\hline Endoscopic ultrasonography & $23(15.1)$ \\
\hline Abdominal MRI & $13(8.6)$ \\
\hline Abdominal ultrasound & $32(21.1)$ \\
\hline Colonoscopy & $8(5.3)$ \\
\hline Small intestine endoscopy & $7(4.6)$ \\
\hline PET-CT & $5(3.3)$ \\
\hline
\end{tabular}

${ }^{a} n=152,30$ patients with co-existing diseases excluded. $C D$, cluster of differentiation; CT, computed tomography; MRI, magnetic resonance imaging; PET, positron emission tomography.

including endoscopic ultrasonography, MRI, abdominal ultrasound, colonoscopy, small intestinal endoscopy (or capsule endoscopy) and PET-CT were used in 23 (15.1\%), 13 (8.6\%), 32 $(21.1 \%), 8(5.3 \%), 7(4.6 \%)$ and $5(3.3 \%)$ patients, respectively
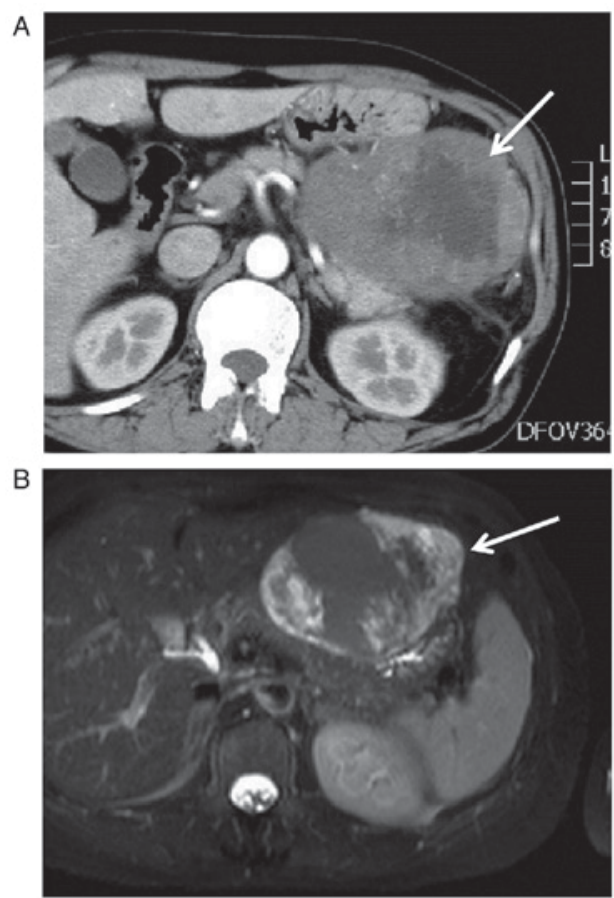

Figure 1. Radiological characteristics of GISTs. (A) Computed tomography scan (arterial phase): The lesion is indicated by the arrow. GIST of the stomach demonstrating a thickening of the gastric wall, with slight to moderate inhomogeneous enhancement following contrast agent injection. Lymph nodes around the mass exhibited reactive hyperplasia. (B) Magnetic resonance imaging (T2-weighted): The lesion is indicated by the arrow. GIST of the stomach sized $\sim 9 \times 7 \mathrm{~cm}$ and exhibiting heterogeneous hyperintense T2 signals. GIST, gastrointestinal stromal tumor.

(Table I and Fig. 1). In addition, 30 patients (16.5\%) were diagnosed with GIST by pathological examination following surgery for other conditions.

The majority of GIST samples were positive for CD117 and DOG1 according to the IHC analysis (98.4 and $98.3 \%$ cases, respectively). In addition, $94.5 \%$ of the samples were positive for CD34. Positive SMA, S-100 protein and desmin expression was also detected in 57.5, 14.2 and $13.9 \%$ of the GISTs, respectively. The $\mathrm{Ki}-67$ index was $0-65 \%$, with a mean of $7 \%$ (Table I and Fig. 2).

The malignant potential of 136 GISTs with data on mitotic rate was evaluated. The distribution of risk groups was 17 $(12.5 \%)$ in the very low-risk, $35(25.7 \%)$ in the low-risk, 31 $(22.8 \%)$ in the intermediate-risk and $53(39.0 \%)$ in the high-risk groups according to the NIH criteria. In addition, 72 cases $(52.9 \%)$ were classified in the benign group, $5(3.68 \%)$ in the malignant potential group and $59(43.4 \%)$ in the malignancy group according to the AFIP criteria. It was also demonstrated that larger GISTs exhibited a higher mitotic rate $(\mathrm{P}<0.001)$. The NIH risk classification of GISTs at different sites was significantly different $(\mathrm{P}=0.006)$, with GISTs in the stomach or duodenum exhibiting a lower risk of malignancy. However, the AFIP risk classification and mitotic rate of GISTs did not differ significantly by primary location $(\mathrm{P}=0.0996$ and $\mathrm{P}=0.1203$, respectively). Based on the symptoms of the 136 patients when they were admitted to Changzheng Hospital, the patients were divided into the asymptomatic GIST group (asymptomatic patients and patients accidentally diagnosed with co-existing disease; $n=46)$ and the symptomatic GIST group $(n=90)$. 


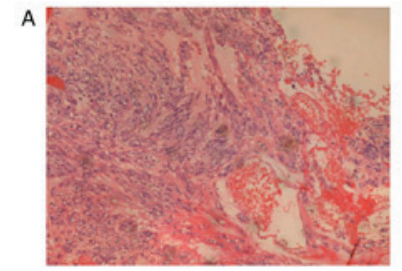

C
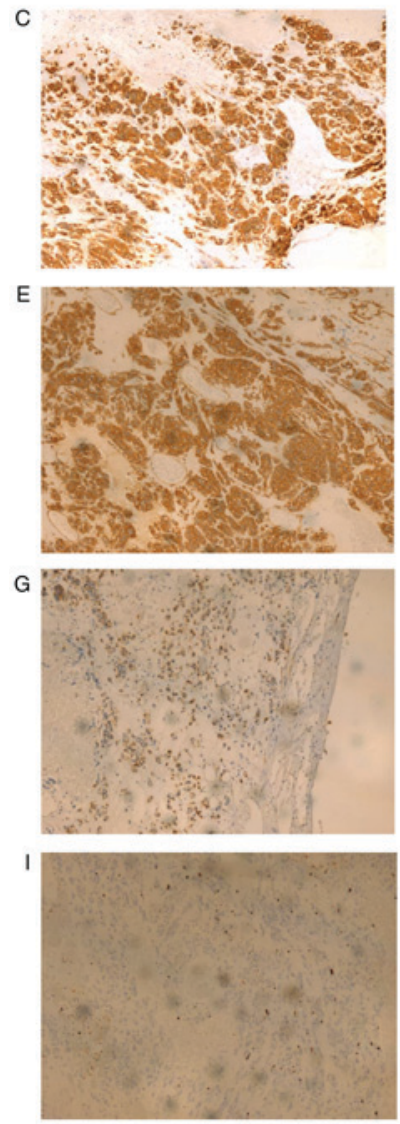
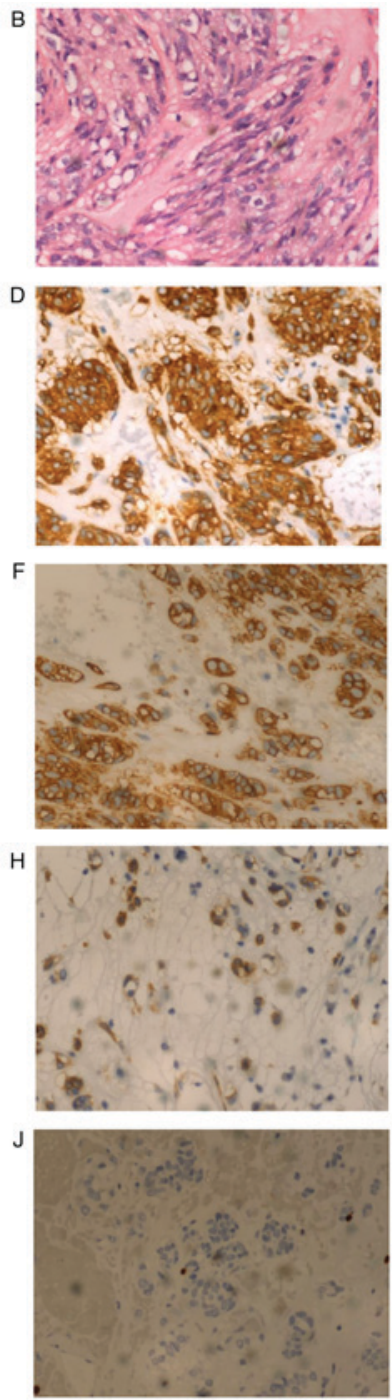

Figure 2. Histological and immunohistochemical characteristics of GISTs. H\&E staining; magnification, (A) x100 and (B) x400. Strong and diffuse staining for CD117; magnification, (C) x100 and (D) x400. Strong and diffuse staining for CD34; magnification, (E) x100 and (F) x400. Staining for DOG1; magnification, $(\mathrm{G}) \times 100$ and $(\mathrm{H}) \times 400$. Staining for Ki-67; magnification, (I) X100 and (J) x400. GIST, gastrointestinal stromal tumor; CD, cluster of differentiation.

Patients in the asymptomatic group had a smaller tumor size $(\mathrm{P}=0.0245)$ and a lower risk of malignancy according to the NIH $(\mathrm{P}=0.0327)$ and AFIP $(\mathrm{P}=0.0198)$ risk classification criteria (Tables II and III).

Treatment. Numerous surgical procedures were performed, including partial gastric resection, total gastric resection, partial intestinal resection, hemicolectomy, sigmoid colon resection, abdomino-perineal rectum resection, pancreatoduodenectomy, distal pancreatectomy and endoscopic submucosal dissection. The choice of surgical procedure was individualized, depending on the tumor location, size and possibility of complete resection. Excluding the 30 patients who were incidentally diagnosed with GIST while treated for other conditions, 125 of the remaining 152 patients underwent radical resection of the primary tumor. Among these patients, 71 underwent open surgical resection; 47 underwent laparoscopic resection; 3 underwent endoscopic surgery; and

4 underwent endoscopic-assisted laparoscopic surgery. An additional 27 patients with unresectable tumors received palliative surgery, including 19 open surgery and 8 laparoscopic surgery. In 24 of the patients, the tumors displayed clear malignant characteristics during surgery, including local invasion and metastasis. Additionally, 5 patients were revealed to have tumor bleeding and ulceration. Imatinib as adjuvant therapy was administered to 15 patients with intermediate-to-high-risk GISTs, including 6 patients with advanced disease. No neoadjuvant imatinib therapy was used in the patients enrolled in the present study.

Survival analysis. Based on the 152 patients without co-existing diseases, the median follow-up time was 48 months (range, 3-81 months), and the 5-year OS and RFS rates were $85.4 \%$ (95\% CI: 79.5-91.3) and 83.8\% (95\% CI: 77.5-90.1), respectively. A total of 21 patients succumbed during the follow-up period as a result of various causes (disease progression, other chronic diseases, including diabetes mellitus, cardiovascular and cerebral disorders, other malignancies and trauma). A total of 4 patients developed metastases in the abdominopelvic cavity and 3 in the liver during follow-up. The results of the univariate analysis of potential prognostic factors are presented in Table IV and Fig. 3. Larger tumor size ( $>10 \mathrm{~cm} ; \mathrm{P}<0.001)$, higher mitotic rate ( $>10 / 50 \mathrm{HPF} ; \mathrm{P}<0.001)$, aggressive behavior, including tumor metastasis or local invasion prior to treatment $(\mathrm{P}<0.001)$ and negative SMA expression $(\mathrm{P}=0.009)$ contributed toward poorer survival of patients with GIST. In addition, non-gastric disease location $(\mathrm{P}<0.001)$, larger tumor size $(>10 \mathrm{~cm} ; \mathrm{P}<0.001)$, higher mitotic rate $(>10 / 50 \mathrm{HPF} ; \mathrm{P}=0.004)$ and aggressive behavior $(\mathrm{P}<0.001)$ were associated with higher risk of recurrence. Patients receiving palliative tumor resection had a significantly shorter survival time $(\mathrm{P}<0.001)$ and a higher risk of recurrence $(\mathrm{P}<0.001)$. When therapeutic factors were included in the multivariate analysis, palliative surgical resection was the only independent risk factor for OS (HR=9.196, 95\% CI: 3.327-25.417, $\mathrm{P}<0.001)$ and RFS $(\mathrm{HR}=16.42,95 \% \mathrm{CI}: 6.065-44.454, \mathrm{P}<0.001)$. If the therapeutic factors were excluded, the multivariate analysis indicated that the mitotic rate $(\mathrm{HR}=3.761,95 \% \mathrm{CI}: 1.288-10.987, \mathrm{P}=0.015)$ and aggressive behavior $(\mathrm{HR}=3.916,95 \% \mathrm{CI}$ : $1.389-11.044$, $\mathrm{P}=0.010)$ were independent risk factors for OS. Non-gastric disease location $(\mathrm{HR}=4.740,95 \% \mathrm{CI}: 1.747-12.857, \mathrm{P}=0.002)$ and aggressive behavior $(\mathrm{HR}=4.009,95 \% \mathrm{CI}$ : $1.538-10.449$, $\mathrm{P}=0.004)$ were independent risk factors for RFS (Table V).

\section{Discussion}

The present retrospective study, based on 182 Chinese patients with GIST, aimed to investigate the clinicopathological and prognostic characteristics of this disease. The results are comparable with those of previous studies in other populations (23-27). The median age of the patients in the present study was 60 years. The stomach was the most common primary site of GISTs, while patients with GIST usually lack specific symptoms. In line with the results of a Japanese study that indicated that GISTs may be incidentally discovered during gastric cancer screening (28), 36 patients in the present study were asymptomatic without co-existing diseases and their GISTs were detected during their annual physical exam. 
Table II. Association between tumor site, tumor size and mitotic rate.

\begin{tabular}{|c|c|c|c|c|}
\hline Factor & $\mathrm{n}$ & 0-5/50 HPF & 5-10/50 HPF & $>10 / 50 \mathrm{HPF}$ \\
\hline \multicolumn{5}{|l|}{ Location } \\
\hline Stomach & 101 & 59 & 33 & 9 \\
\hline Duodenum & 9 & 7 & 1 & 1 \\
\hline Jejunum and ileum & 21 & 10 & 7 & 4 \\
\hline Colon, rectum and anus & 4 & 0 & 3 & 1 \\
\hline Omentum & 1 & 1 & 0 & 0 \\
\hline \multicolumn{5}{|l|}{ Tumor size, cm } \\
\hline $0-2$ & 15 & 14 & 1 & 0 \\
\hline $2-5$ & 59 & 36 & 18 & 5 \\
\hline $5-10$ & 40 & 22 & 16 & 2 \\
\hline$\geq 10$ & 22 & 5 & 9 & 8 \\
\hline Total & 136 & 77 & 44 & 15 \\
\hline
\end{tabular}

Table III. Association between symptoms, tumor site and NIH or AFIP risk classification criteria.

\begin{tabular}{|c|c|c|c|c|c|c|c|c|}
\hline \multirow[b]{2}{*}{ Factor } & \multirow[b]{2}{*}{$\mathrm{n}$} & \multicolumn{4}{|c|}{$\mathrm{NIH}$} & \multicolumn{3}{|c|}{ AFIP } \\
\hline & & Very low & Low & Middle & High & Benign & Malignant potential & Malignancy \\
\hline \multicolumn{9}{|l|}{ Location } \\
\hline Stomach & 101 & 16 & 25 & 29 & 31 & 56 & 3 & 42 \\
\hline Duodenum & 9 & 1 & 5 & 1 & 2 & 7 & 0 & 2 \\
\hline Jejunum and ileum & 21 & 0 & 5 & 0 & 16 & 8 & 1 & 12 \\
\hline Colon, rectum and anus & 4 & 0 & 0 & 1 & 3 & 0 & 1 & 3 \\
\hline Omentum & 1 & 0 & 0 & 0 & 1 & 1 & 0 & 0 \\
\hline \multicolumn{9}{|l|}{ Symptoms } \\
\hline Asymptomatic & 46 & 8 & 13 & 14 & 11 & 31 & 1 & 14 \\
\hline Symptomatic & 90 & 9 & 22 & 17 & 42 & 41 & 4 & 45 \\
\hline Total & 136 & 17 & 35 & 31 & 53 & 72 & 5 & 59 \\
\hline
\end{tabular}

NIH, National Institutes of Health; AFIP, Armed Forces Institute of Pathology.

Radical tumor resection is the most important factor affecting patient prognosis. Furthermore, non-gastric disease location, higher mitotic rate and tumor metastasis or local invasion prior to treatment were revealed to be predictors of a poor prognosis.

For GIST patients with clinical symptoms and those with incidentally detected tumors during physical examination, further radiological and endoscopic examinations are required (29). In the present study, 52 patients were diagnosed with GIST by gastroscopy and 65 by abdominal CT. Gastroscopic and endoscopic ultrasonography can detect mostly intramural tumors and enable acquisition of cytological or histological samples, while the use of endoscopy is limited when evaluating metastasis outside the digestive tract (30). Abdominal CT can scan the whole abdomen and is able to detect small lesions, providing valuable information on the size, morphology, aggressiveness and metastasis of the tumors $(31,32)$. Abdominal MRI and PET-CT also have high diagnostic sensibility, particularly in intestinal GISTs or
EGISTs, and were used in 13 and 5 patients, respectively. MRI is affected by peristalsis of the gastrointestinal tract, which limits its applicability, although it has been reported that, for lesions of the rectum and liver, MRI may offer more detailed images compared with CT (33). PET-CT is applied for evaluating tumor metastasis and response following the initiation of targeted therapy (34). Among patients with intestinal GISTs, the diagnosis of 7 patients in the present study series was confirmed by small intestinal endoscopy or capsule endoscopy, as their tumors were relatively difficult to diagnose.

There are no standard criteria for assessing the aggressive behavior and predicting the clinical prognosis of GISTs, although the NIH and AFIP criteria are widely recommended (35). It is commonly accepted that all GISTs are considered to have malignant potential (36). Through multivariate analysis, higher mitotic rate and tumor metastasis or local invasion prior to treatment were revealed to be associated with poor survival in GIST patients, and non-gastric 
Table IV. Univariate analysis of prognostic factors for OS and RFS in 152 GIST patients.

\begin{tabular}{|c|c|c|c|c|c|}
\hline \multirow[b]{2}{*}{ Group } & \multirow[b]{2}{*}{$\mathrm{N}$} & \multicolumn{2}{|c|}{ OS } & \multicolumn{2}{|c|}{ RFS } \\
\hline & & 5-year OS & P-value & 5-year RFS & P-value \\
\hline \multicolumn{6}{|l|}{ Sex } \\
\hline Male & 69 & 84.0 & \multirow[t]{2}{*}{0.724} & 81.8 & \multirow[t]{2}{*}{0.361} \\
\hline Female & 83 & 86.5 & & 85.5 & \\
\hline \multicolumn{6}{|l|}{ Age (years) } \\
\hline$\leq 60$ & 83 & 88.5 & \multirow[t]{2}{*}{0.177} & 87.1 & \multirow[t]{2}{*}{0.360} \\
\hline$>60$ & 69 & 81.3 & & 79.5 & \\
\hline \multicolumn{6}{|l|}{ Disease location } \\
\hline Gastric & 106 & 87.8 & \multirow[t]{2}{*}{0.192} & 90.8 & \multirow[t]{2}{*}{$<0.001^{\mathrm{a}}$} \\
\hline Non-gastric & 46 & 79.9 & & 68.5 & \\
\hline \multicolumn{6}{|l|}{ Tumor size (cm) } \\
\hline$\leq 10$ & 129 & 91.1 & \multirow[t]{2}{*}{$<0.001^{\mathrm{a}}$} & 87.6 & \multirow[t]{2}{*}{$<0.001^{\mathrm{a}}$} \\
\hline$>10$ & 23 & 55.3 & & 60.1 & \\
\hline \multicolumn{6}{|l|}{ Mitotic rate (/50 HPF) } \\
\hline$\leq 10$ & 107 & 89.3 & \multirow[t]{2}{*}{$<0.001^{\mathrm{a}}$} & 87.3 & \multirow[t]{2}{*}{$0.004^{\mathrm{a}}$} \\
\hline$>10$ & 14 & 54.5 & & 58.9 & \\
\hline \multicolumn{6}{|c|}{ Metastatic disease or local invasion } \\
\hline Yes & 24 & 49.0 & \multirow[t]{2}{*}{$<0.001^{\mathrm{a}}$} & 44.9 & \multirow[t]{2}{*}{$<0.001^{\mathrm{a}}$} \\
\hline No & 128 & 91.9 & & 90.2 & \\
\hline \multicolumn{6}{|l|}{ Ulceration } \\
\hline Yes & 5 & 80.0 & \multirow[t]{2}{*}{0.664} & 80.0 & 0.120 \\
\hline No & 147 & 85.5 & & 84.0 & \\
\hline Desmin & & & & & \\
\hline Positive & 19 & 82.6 & 0.841 & 85.9 & 0.539 \\
\hline Negative & 130 & 85.7 & & 83.3 & \\
\hline S-100 & & & & & \\
\hline Positive & 23 & 85.6 & 0.907 & 95.5 & 0.132 \\
\hline Negative & 126 & 86.0 & & 82.1 & \\
\hline SMA & & & & & \\
\hline Positive & 89 & 91.4 & $0.009^{\mathrm{a}}$ & 85.4 & 0.448 \\
\hline Negative & 58 & 76.7 & & 80.3 & \\
\hline Symptom & & & & & \\
\hline Asymptomatic & 36 & 91.2 & 0.301 & 93.0 & 0.077 \\
\hline Symptomatic & 116 & 83.6 & & 81.0 & \\
\hline Resection margin & & & & & \\
\hline Radical & 125 & 93.4 & $<0.001^{\mathrm{a}}$ & 94.4 & $<0.001^{\mathrm{a}}$ \\
\hline Palliative & 27 & 46.6 & & 28.4 & \\
\hline Surgical procedures & & & & & \\
\hline Open & 90 & 82.6 & 0.232 & 82.8 & 0.587 \\
\hline Minimally invasive & 62 & 89.7 & & 85.0 & \\
\hline Imatinib therapy ${ }^{\mathrm{b}}$ & & & & & \\
\hline Yes & 15 & 86.2 & 0.388 & 54.3 & 0.052 \\
\hline No & 71 & 77.0 & & 83.0 & \\
\hline
\end{tabular}

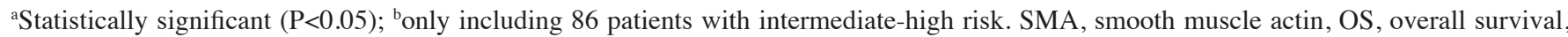
RFS, relapse-free survival; $\mathrm{N}$, number. 
Table V. Multivariate analysis of prognostic factors for OS and RFS in 152 patients with gastrointestinal stromal tumors (therapeutic factors excluded).

\begin{tabular}{|c|c|c|c|c|c|c|}
\hline \multirow[b]{2}{*}{ Factor } & \multicolumn{3}{|c|}{ OS } & \multicolumn{3}{|c|}{ RFS } \\
\hline & HR & $95 \% \mathrm{CI}$ & P-value & HR & $95 \%$ CI & P-value \\
\hline Disease location (gastric vs. non-gastric) & - & - & - & 4.740 & $1.747-12.857$ & $0.002^{\mathrm{a}}$ \\
\hline Mitotic rate ( $\leq 10 / 50 \mathrm{HPF}$ vs. $>10 / 50 \mathrm{HPF})$ & 3.761 & $1.288-10.987$ & $0.015^{\mathrm{a}}$ & - & - & - \\
\hline Metastatic disease or adjacent involvement (no vs. yes) & 3.916 & $1.389-11.044$ & $0.010^{\mathrm{a}}$ & 4.009 & $1.538-10.449$ & $0.004^{\mathrm{a}}$ \\
\hline
\end{tabular}

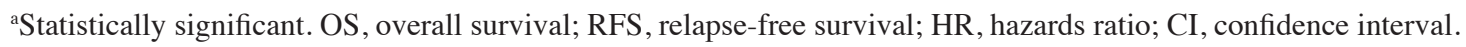
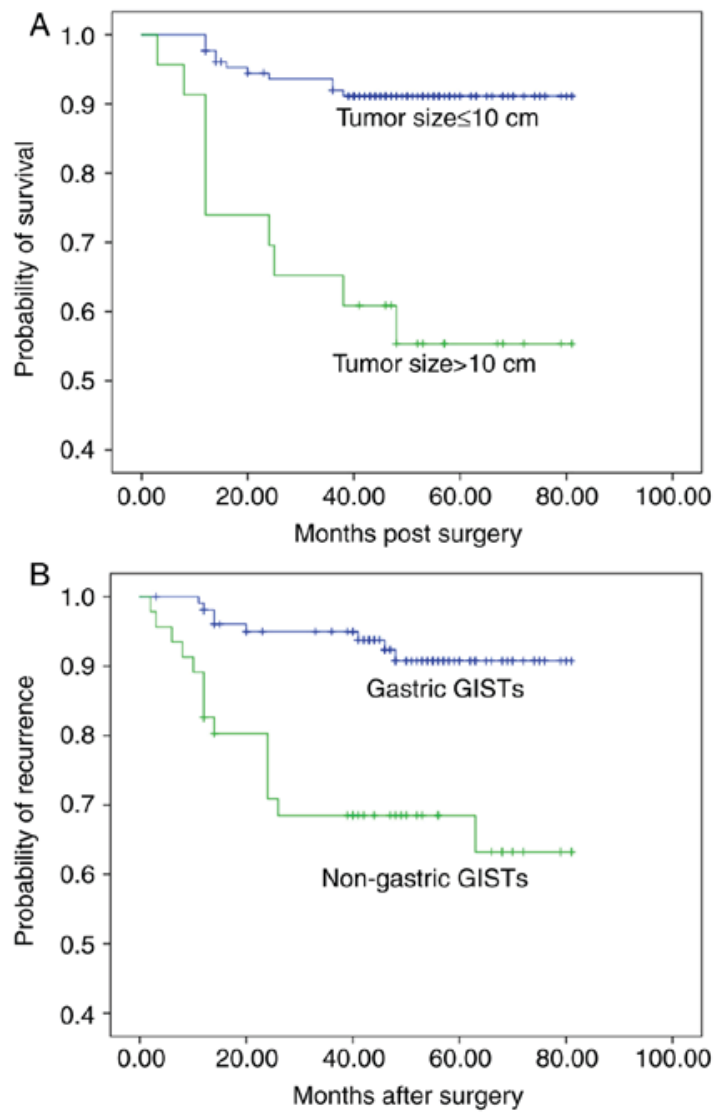

Figure 3. Survival analysis of 152 patients. (A) OS according to tumor size $(\mathrm{P}<0.001)$. (B) RFS according to tumor location $(\mathrm{P}<0.001)$. OS, overall survival; RFS, relapse-free survival.

Miettinen and Lasota (37) also demonstrated that small intestinal GISTs behave more aggressively than gastric GISTs, and small intestinal GISTs tend to be larger and more advanced at diagnosis. Liu et al (35) suggested that gastrointestinal bleeding is a prognostic factor. However, preoperative symptoms did not appear to affect the outcome of patients with GIST in our analysis. Large tumor size is considered to be a prognostic factor in the NIH and AFIP risk classification criteria. However, it failed to be an independent risk factor in the present study. Notably, patients with SMA-negative tumors exhibited a shorter survival time. Similarly, Demir et al (38) reported that patients with SMA-positive GISTs tended to survive longer and had significantly longer disease-free survival (DFS) times than the SMA-negative cases. Fujimoto et al (39) reported no association between SMA IHC analysis and the prognosis of patients with GIST. However, Bertin et al (40) reported that SMA positivity is significantly associated with a lower 5 -year survival rate (39 vs. 100\%). Differences in the selected population, tumor location, disease stage and treatment between these studies may affect these conclusions.

GISTs are usually asymptomatic until they reach a large size, at which point they may cause non-specific symptoms or be detected as a palpable mass (41). Compared with patients diagnosed with clinical symptoms, asymptomatic patients are considered to have early-stage disease. A total of 46 patients in the present study were asymptomatic or accidentally diagnosed with co-existing disease, and these GISTs were smaller in size and exhibited a lower risk based on the NIH and AFIP risk classification criteria, although no significant effect on OS and RFS was observed. A study by Yamamoto et al (28) reported that GISTs are incidentally observed during gastric cancer screenings in Japan. Over half of these patients are asymptomatic and have smaller tumors $(\mathrm{P}<0.001)$ and lower recurrence rates $(\mathrm{P}=0.017)$, compared with symptomatic patients. Therefore, the Japanese gastric cancer screening system contributes toward the early detection of gastric GISTs and favorable treatment outcomes by identifying asymptomatic patients. Scherubl et al (30) demonstrated that early asymptomatic GISTs have an excellent GIST-specific prognosis. The results of the present study also suggested that detecting GISTs at an early stage may improve patient outcome.

For resectable localized GISTs, radical surgery is the standard and first choice of treatment (42). Radical tumor resection significantly improved survival and reduced tumor recurrence, in univariate or multivariate analyses. Different surgical approaches, including open and laparoscopic surgery and endoscopic procedures, were performed in the present study. No statistically significant difference was observed in OS or RFS among these surgical strategies. A number of studies have been performed comparing the effect of minimally invasive and open surgery in the treatment of GISTs (42-44). It is generally accepted that minimally invasive surgery has similar or even superior perioperative outcomes, without compromising the oncological outcomes; it may also be safely used for larger tumors or tumors located in unfavorable sites. Imatinib serves an important role in the treatment of advanced GISTs and in the adjuvant setting, reducing the risk of recurrence and metastasis $(16,45)$. In the 
present study, not all the patients with intermediate-to-high risk GIST received imatinib as adjuvant therapy. However, there was no observed improvement in OS and RFS in the 15 patients who were administered adjuvant imatinib therapy. One possible reason may be that the selection of candidates for adjuvant therapy was not standardized and the sample size was limited. Imatinib was also recommended to patients receiving palliative surgery and those with disease progression. Advanced GISTs will inevitably progress and reduce the OS and RFS rates (16).

GISTs are widely considered to have a low risk of lymph node metastasis; therefore, lymphadenectomy is not deemed necessary during surgical resection $(46,47)$. However, GIST cases with lymph node metastasis have been reported. Tashiro et al (48) and Shafizad et al (49) reported two cases of lymph node involvement in gastric GISTs. In addition, Gong et al (50) reported that 6 of $29(20.7 \%)$ patients with GIST were revealed to have lymph node metastasis on PET-CT imaging. In the present study, lymph node metastasis was detected in only 1 patient with a history of intestinal GIST resection 2 years prior. Palliative surgery was performed and two main masses were removed from the small intestine. Tumor ulceration and bleeding were observed intraoperatively, and liver, peritoneal and pelvic cavity metastasis were confirmed. All 7 mesenteric lymph nodes resected during surgery were positive. Despite these reports, however, GISTs rarely metastasize to the lymph nodes, and regional lymph node resection is of unproven value (16).

There were certain limitations to the present study: The design of the study was retrospective; the selection of surgical approach and adjuvant therapy were not standardized; and the use of imatinib as an adjuvant therapy was limited to 15 patients with a potential selection bias; therefore, the benefit of using imatinib as adjuvant therapy cannot be evaluated based on this study. In summary, the present study updated the clinicopathological and immunophenotypic characteristics of GISTs in mainland China. Asymptomatic GISTs may be of smaller size and have a lower risk of malignancy according to the NIH and AFIP risk classification criteria. Clinical and immunohistochemical results were used for survival analysis, and positive SMA was associated with an improved survival in univariate analysis. Higher mitotic rate and tumor metastasis or local invasion prior to treatment were revealed to be independent risk factors for a poor OS, whereas non-gastric disease location and aggressive behavior were independent risk factors for a poor RFS. Large tumor size, a prognostic factor in the NIH and AFIP risk classification criteria, failed to reveal significant impact on OS and RFS in multivariate analysis. The present study may aid clinicians with an improved understanding of the diagnosis and treatment of GISTs.

\section{Acknowledgements}

Not applicable.

\section{Funding}

This study was supported by the Project of Innovation Incubation Base of Second Military Medical University (grant no. FH2016183).

\section{Availability of data and materials}

All data generated and/or analyzed during this study are included in this published article.

\section{Authors' contributions}

YML analyzed and interpreted the patient data regarding GIST disease, performed statistical analysis and wrote the manuscript. WJC recorded the follow-up information, performed statistical analysis and wrote the manuscript. ZWB analyzed and interpreted the data and critically reviewed the manuscript. YDK designed the study and the quality control of data and algorithms, and reviewed the manuscript. All authors read and approved the final manuscript.

\section{Ethics approval and consent to participate}

The present retrospective study was approved by Changzheng Hospital Medical Committee. Written informed consent was obtained from all participating patients.

\section{Patient consent for publication}

The patient or parent, guardian or next of kin provided written informed consent for the publication of any associated data and accompanying images. All identifying information was removed.

\section{Competing interests}

The authors declare that they have no competing interests.

\section{References}

1. Mazur MT and Clark HB: Gastric stromal tumors. Reappraisal of histogenesis. Am J Surg Pathol 7: 507-519, 1983.

2. Goettsch WG, Bos SD, Breekveldt-Postma N, Casparie M, Herings RM and Hogendoorn PC: Incidence of gastrointestinal stromal tumours is underestimated: Results of a nation-wide study. Eur J Cancer 41: 2868-2872, 2005.

3. Wang M, XU J, Zhang Y, Tu L, Qiu WQ, Wang CJ, Shen YY, Liu Q and Cao H: Gastrointestinal stromal tumor: 15-years experience in a single center. BMC Surg 14: 93, 2014.

4. Pisters PW, Blanke CD, von Mehren M, Picus J, Sirulnik A, Stealey E, Trent JC and reGISTry Steering Committee: A USA registry of gastrointestinal stromal tumor patients: Changes in practice over time and differences between community and academic practices. Ann Oncol 22: 2523-2529, 2011.

5. Miettinen M, Monihan JM, Sarlomo-Rikala M, Kovatich AJ, Carr NJ, Emory TS and Sobin LH: Gastrointestinal stromal tumors/smooth muscle tumors (GISTs) primary in the omentum and mesentery: Clinicopathologic and immunohistochemical study of 26 cases. Am J Surg Pathol 23: 1109-1118, 1999.

6. Miettinen M, Makhlouf H, Sobin LH and Lasota J: Gastrointestinal stromal tumors of the jejunum and ileum: A clinicopathologic, immunohistochemical, and molecular genetic study of 906 cases before imatinib with long-term follow-up. Am J Surg Pathol 30: 477-489, 2006.

7. Zhao $\mathrm{X}$ and Yue C: Gastrointestinal stromal tumor. J Gastrointest Oncol 3: 189-208, 2012.

8. Blay JY, Shen L, Kang YK, Rutkowski P, Qin S, Nosov D, Wan D, Trent J, Srimuninnimit V, Pápai Z, et al: Nilotinib versus imatinib as first-line therapy for patients with unresectable or metastatic gastrointestinal stromal tumours (ENESTg1): A randomised phase 3 trial. Lancet Oncol 16: 550-560, 2015. 
9. Cassier PA, Ducimetiere F, Lurkin A, Ranchère-Vince D, Scoazec JY, Bringuier PP, Decouvelaere AV, Méeus P, Cellier D, Blay JY and Ray-Coquard I: A prospective epidemiological study of new incident GISTs during two consecutive years in rhone alpes region: Incidence and molecular distribution of GIST in a european region. Br J Cancer 103: 165-170, 2010.

10. Joensuu H: Risk stratification of patients diagnosed with gastrointestinal stromal tumor. Hum Pathol 39: 1411-1419, 2008.

11. Goh BK, Chow PK, Yap WM, Kesavan SM, Song IC, Paul PG, Ooi BS, Chung YF and Wong WK: Which is the optimal risk stratification system for surgically treated localized primary GIST? Comparison of three contemporary prognostic criteria in 171 tumors and a proposal for a modified armed forces institute of pathology risk criteria. Ann Surg Oncol 15: 2153-2163, 2008.

12. Belfiori G, Sartelli M, Cardinali L, Tranà C, Bracci R, Gesuita R and Marmorale C: Risk stratification systems for surgically treated localized primary gastrointestinal stromal tumors (GIST). Review of literature and comparison of the three prognostic criteria: MSKCC nomogramm, NIH-fletcher and AFIP-miettinen. Ann Ital Chir 86: 219-227, 2015.

13. Wang YP, Li YI and Song C: Clinicopathological features and prognosis of small gastrointestinal stromal tumors outside the stomach. Oncol Lett 10: 2723-2730, 2015.

14. Zhang P, Deng R, Liu K, Shuai XM, Bai J, Chang WL, Gao JB, Cai KL, Wang GB and Tao KX: Clinicopathologic features and prognosis of primary gastrointestinal stromal tumor patients under 35 years of age: A 10-year retrospective study. J Surg Oncol 114: 977-981, 2016

15. Liu X, Qiu H, Zhang P, Feng X, Chen T, Li Y, Tao K, Li G, Sun X, Zhou Z and China Gastrointestinal Stromal Tumor Study Group (CN-GIST): Ki-67 labeling index may be a promising indicator to identify 'very high risk' gastrointestinal stromal tumor: A multicenter retrospective study of 1022 patients. Hum Pathol 74 $17-24,2018$.

16. Cao H, Zhang Y, Wang M, Shen DP, Sheng ZY, Ni XZ, Wu ZY, Liu Q, Shen YY and Song YY: Prognostic analysis of patients with gastrointestinal stromal tumors: A single unit experience with surgical treatment of primary disease. Chin Med J (Engl) 123: 131-136, 2010.

17. Mrowiec S, Jabłońska B, Liszka L, Pajak J, Leidgens M, Szydło R, Sandecka A and Lampe P: Prognostic factors for survival post surgery for patients with gastrointestinal stromal tumors. Eur Surg Res 48: 3-9, 2012

18. Wang X, Li WQ, Yan HZ, Li YM, He J, Liu HM and Yu HY: Alveolar adenoma combined with multifocal cysts: Case report and literature review. J Int Med Res 41: 895-906, 2013.

19. Badic B, Gancel Ch, Thereaux J, Joumond A, Bail JP, Meunier B and Sulpice L: Surgical and oncological long term outcomes of gastrointestinal stromal tumors (GIST) resection-retrospective cohort study. Int J Surg 53: 257-261, 2018

20. Tan Y, Tan L, Lu J, Huo J and Liu D: Endoscopic resection of gastric gastrointestinal stromal tumors. Transl Gastroenterol Hepatol 2: 115, 2017.

21. Koh YX and Goh B: Minimally invasive surgery for gastric gastrointestinal stromal tumors. Transl Gastroenterol Hepatol 2: $108,2017$.

22. Ford SJ and Gronchi A: Indications for surgery in advanced/metastatic GIST. Eur J Cancer 63: 154-167, 2016.

23. Tran T, Davila Ja and El-serag HB: The epidemiology of malignant gastrointestinal stromal tumors: An analysis of 1,458 cases from 1992 to 2000 . Am J Gastroenterol 100: $162-168,2005$

24. Tryggvason G, Gislason HG, Magnusson MK and Jonasson JG: Gastrointestinal stromal tumors in Iceland, 1990-2003: The icelandic GIST study, a population-based incidence and pathologic risk stratification study. Int J Cancer 117: 289-293, 2005.

25. Rubio J, Marcos-Gragera R, Ortiz MR, Miró J, Vilardell L, Gironès J, Hernandez-Yagüe X, Codina-Cazador A, Bernadó L, Izquierdo A and Colomer R: Population-based incidence and survival of gastrointestinal stromal tumours (GIST) in girona, spain. Eur J Cancer 43: 144-148, 2007

26. Mucciarini C, Rossi G, Bertolini F, Valli R, Cirilli C, Rashid I, Marcheselli L, Luppi G and Federico M: Incidence and clinicopathologic features of gastrointestinal stromal tumors. A population-based study. BMC Cancer 7: 230, 2007.

27. Chiang NJ, Chen LT, Tsai CR and Chang JS: The epidemiology of gastrointestinal stromal tumors in taiwan, 1998-2008: A nation-wide cancer registry-based study. BMC Cancer 14: 102, 2014.
28. Yamamoto K, Tsujinaka T, Takahashi T, Sato S, Nishiguchi Y, Nakashima Y, Muguruma K, Hirota S and Nishida T: Impact of the Japanese gastric cancer screening system on treatment outcomes in gastric gastrointestinal stromal tumor (GIST): An analysis based on the GIST registry. Ann Surg Oncol 22: 232-239, 2015

29. Sandrasegaran K, Rajesh A, Rydberg J, Rushing DA, Akisik FM and Henley JD: Gastrointestinal stromal tumors: Clinical, radiologic, and pathologic features. AJR Am J Roentgenol 184: 803-811, 2005 .

30. Scherubl H, Faiss S, Knoefel WT and Wardelmann E: Management of early asymptomatic gastrointestinal stromal tumors of the stomach. World J Gastrointest Endosc 6: 266-271, 2014.

31. Horton KM, Juluru K, Montogomery E and Fishman EK: Computed tomography imaging of gastrointestinal stromal tumors with pathology correlation. J Comput Assist Tomogr 28: 811-817, 2004.

32. Shi Z and Zhuang Q: Computed tomography imaging characteristics of synchronous gastrointestinal stromal tumors in patients with gastric cancer and correlation with clinicopathological findings. Mol Clin Oncol 3: 1311-1314, 2015.

33. Lamba G, Gupta R, Lee B, Ambrale S and Liu D: Current management and prognostic features for gastrointestinal stromal tumor (GIST). Exp Hematol Oncol 1: 14, 2012.

34. Tokumoto N, Tanabe K, Misumi T, Fujikuni N, Suzuki T and Ohdan H: The usefulness of preoperative 18FDG positronemission tomography and computed tomography for predicting the malignant potential of gastrointestinal stromal tumors. Dig Surg 31: 79-86, 2014.

35. Liu Q, Li Y, Dong M, Kong F and Dong Q: Gastrointestinal bleeding is an independent risk factor for poor prognosis in GIST patients. Biomed Res Int 2017: 7152406, 2017.

36. Yamamoto $\mathrm{H}$ and Oda $\mathrm{Y}$ : Gastrointestinal stromal tumor: Recent advances in pathology and genetics. Pathol Int 65: 9-18, 2015.

37. Miettinen $\mathrm{M}$ and Lasota J: Gastrointestinal stromal tumors: Review on morphology, molecular pathology, prognosis, and differential diagnosis. Arch Pathol Lab Med 130: 1466-1478, 2006.

38. Demir L, Ekinci N, Erten C, Kucukzeybek Y, Alacacioglu A, Somali I, Can A, Dirican A, Bayoglu V, Akyol M, et al: Does immunohistochemistry provide additional prognostic data in gastrointestinal stromal tumors? Asian Pac J Cancer Prev 14: 4751-4758, 2013.

39. Fujimoto Y, Nakanishi Y, Yoshimura K and Shimoda T: Clinicopathologic study of primary malignant gastrointestinal stromal tumor of the stomach, with special reference to prognostic factors: Analysis of results in 140 surgically resected patients. Gastric Cancer 6: 39-48, 2003

40. Bertin M, Angriman I, Scarpa M, Mencarelli R, Ranzato R, Ruffolo C, Polese L, Iacobone M and D'Amico DF: Prognosis of gastrointestinal stromal tumors. Hepatogastroenterology 54: 124-128, 2007.

41. Dematteo RP, Heinrich MC, El-Rifai WM and Demetri G: Clinical management of gastrointestinal stromal tumors: Before and after STI-571. Hum Pathol 33: 466-477, 2002.

42. Novitsky YW, Kercher KW, Sing RF and Heniford BT: Long-term outcomes of laparoscopic resection of gastric gastrointestinal stromal tumors. Ann Surg 243: 738-745, 745-747, 2006.

43. Lin J, Huang C, Zheng C, Li P, Xie J, Wang J and Lu J: Laparoscopic versus open gastric resection for larger than $5 \mathrm{~cm}$ primary gastric gastrointestinal stromal tumors (GIST): A size-matched comparison. Surg Endosc 28: 2577-2583, 2014.

44. Liao GQ, Chen T, Qi XL, Hu YF, Liu H, Yu J and Li GX: Laparoscopic management of gastric gastrointestinal stromal tumors: A retrospective 10-year single-center experience. World J Gastroenterol 23: 3522-3529, 2017.

45. Blay JY, Le Cesne A, Ray-Coquard I, Bui B, Duffaud F, Delbaldo C, Adenis A, Viens P, Rios M, Bompas E, et al: Prospective multicentric randomized phase III study of imatinib in patients with advanced gastrointestinal stromal tumors comparing interruption versus continuation of treatment beyond 1 year: The french sarcoma group. J Clin Oncol 25: 1107-1113, 2007. 
46. Papalambros A, Petrou A, Brennan N, Bramis K, Felekouras E and Papalambros E: GIST suture-line recurrence at a gastrojejunal anastomosis 8 years after gastrectomy: Can GIST ever be described as truly benign? A case report. World J Surg Oncol 8: 90,2010.

47. Demetri GD, Benjamin RS, Blanke CD, Blay JY, Casali P, Choi H, Corless CL, Debiec-Rychter M, DeMatteo RP, Ettinger DS, et al: NCCN task force report: Management of patients with gastrointestinal stromal tumor (GIST)-update of the NCCN clinical practice guidelines. J Natl Compr Canc Netw 2 (Suppl 5): S1-S29, S30, 2007.

48. Tashiro T, Hasegawa T, Omatsu M, Sekine S, Shimoda T and Katai H: Gastrointestinal stromal tumour of the stomach showing lymph node metastases. Histopathology 47: 438-439, 2005 .
49. Shafizad A, Mohammadianpanah M, Nasrolahi H, Mokhtari M and Mousavi SA: Lymph node metastasis in gastrointestinal stromal tumor (GIST): To report a case. Iran J Cancer Prev 7: 171-174, 2014.

50. Gong N, Wong CS and Chu YC: Is lymph node metastasis a common feature of gastrointestinal stromal tumor? PET/CT correlation. Clin Nucl Med 36: 678-682,2011. International (CC BY-NC-ND 4.0) License. 